$\underline{\xi}=\mathbf{Z}$

\title{
Matter exhibiting absolute rest and the origin of the solar system
}

\author{
D.S. Robertson * \\ Organization. Private. Address: 205, Pickersleigh Road, Malvern, Worcestershire, England, WR14 2QS \\ *Corresponding author E-mail: danielstewartrobertson@gmail.com
}

\begin{abstract}
The development of a volume of space containing matter which exhibits no movement of any kind is described. This is identified as matter in a state of Absolute Rest as envisaged by Newton. The proper-ties of matter in this state are described. The induction of the change from motion to rest and the re-verse are detailed and the latter applied to the origin of the Sun and the development of the Solar Sys-tem.
\end{abstract}

Keywords: Absolute Zero; Absolute Rest.

\section{Introduction}

At present there are fifteen different proposals concerning the formation of the Solar system each of which involves the origin, nature and properties of the Sun. A possible structure of the Sun and other stars has been derived from basic physical principles supported by measured properties such as the luminosity, radius, composition, asteroseismology and age of the Sun or star. The last value is obtained indirectly from studies of meteorites. The structure of the Sun is considered to be as a spherical volume of mainly hydrogen gas in varying states of ionisation. The entire mass is restrained in the globular form by the force of gravity. The origin of this force is unknown. The hydrogen in a central core is assumed to exist as a completely ionised highly compressed plasma with an assigned a temperature of the order of $1.0 \times 10^{15}$ Kelvin and a density of the order of $150 \mathrm{gms} \mathrm{ml}^{-1}$. The energy released by the Sun is considered to be generated entirely by nuclear fusion of hydrogen producing helium. Under these conditions the present composition of the Sun is estimated as $75 \%$ hydrogen and $23 \%$ helium plus approximately $2 \%$ of other elements. The last group is considered to have been partially acquired from space and partially formed in the Sun. From centre to the periphery the temperature and density are proposed as decreasing with an apparent temperature rise to $2 \times 10^{6}$ Kelvin at the outer surface. No explanation is available for the latter conclusion. The hydrogen present in the Sun is advanced as having originated from one of two concepts concerning the origin of the Universe. The first of these concepts considers that nuclear reaction synthesis of the known elements occurs in the Sun and stars thereby accounting for the observed temperatures of these bodies [1]. The formed elements are progressively dispersed to be used in nuclear reactions in regions remote from the origin. These processes lead to a Universe to be without beginning or end in which stars such as the Sun form and disappear endlessly. The second considers the origin of the Universe to have occurred 13.8 billion years ago at a single point from which all space, time, matter and radiation plus the laws governing these came into being from a state of non-existence [2]. By the second concept the process of forming the Sun is considered as having commenced 4.6 billion years ago. The energy radiated from the latter is taken as progressively increasing from the date of origin. It has been advanced that at the date living forms appeared on Earth, that is, approximately 3 billion years before the present date, the energy emitted by the Sun would have been insufficient to initiate this process. The origin of the planets of the Solar System has been the subject of numerous proposals chief among which is the concept that the force of gravity exerted by the formed Sun gathered material into orbit around this body and from this material planets evolved [3].

The above description of the origin of the Sun and planets of the Solar system involves two sets of concepts concerning the organisation of natural phenomena. The first is the description of the structure of atoms and the origin of radiation of all wavelengths [4]. The second group concerns the relative motion of bodies of matter with respect to themselves and radiation [5]. In the first case radiation is considered to originate with the motion of bodies of matter composing atoms. In the second case it is assumed that radiation of any wavelength moves with a fixed velocity under conditions where matter does not exist. This produces the conclusion that bodies of matter progressing towards motion at this fixed velocity exhibit a progressive increase in mass. It is also concluded that the same bodies experience a progressive change in the duration of any period of time. A consequence of the latter is that bodies of matter exhibit rest mass. Such a mass would involve the cessation of radiation emission through arrest of the motions of matter associated with atoms which produce radiation. An extension of the second concept leads the conclusion that the attractive force of gravity associated with a body can attain a value which completely suppresses any emission of radiation [6]. The ceasing of atom motions is also a consequence the gravity suppression of radiation. Atom structures as presently envisaged are electrically neutral and each atom presents a negative atmosphere to an approaching atom which prevents mutual entry and entanglement of two atoms. Gravitational suppression of the emission of radiation implies that the force of gravity overcomes the Coulombic repulsion of the negative atom atmospheres. At the approach to contact the latter force approaches a value of infinity requiring that force gravity develops the same value. In the latter instance no mechanism for such a change has been described. The velocity of light (c) is the multiple of wavelength times frequency. As the value of wavelength increases the corresponding value of frequency decreases in order that the multiple representing velocity remains constant. Under a constant value of $\mathrm{c}$ this leads the conclusion that 
the value of $t$ in the equation for motion of light $\left(x^{2}+y^{2}+z^{2}+\right.$ $\left.c^{2} t^{2}=0\right)$ is variable Frequency equals the inverse of the period. Period has the units of time and substitution of period in the the final term of the above equation removes time. This means that time is invariant and that the velocity of light is not constant. Light is produced by the motion of matter, travels through and with matter and cannot exist or travel in the absence of matter. Time is defined as a measure of change and any selected units of time are constant. The variation of values for particular units of time would produce a difference of the time periods required to give rise to two or more identical changes. This is not observed.

\section{The development of matter in the state of absolute rest}

The mean molecular velocity of gas molecules derived from the Maxwell distribution is a function of temperature such that this velocity value becomes zero at zero temperature. Identifying zero temperature as Absolute Zero gives rise to the condition of zero motion of molecules and atoms through a given volume of space at this temperature. Radiation emission is advanced as originating with motion of atom components. The emission of radiation is described by the Stephan-Boltzmann law. This relationship is also linked to temperature such that when the latter becomes zero the emission of radiation ceases. Identifying zero temperature as Absolute Zero the consequence is that the motion of components of atoms also ceases. It is proposed that a body of matter at Absolute Rest originates in a volume of space at the centre of an intergalactic hydrogen gas cloud. The interior of this volume is shielded from all sources energy by the surrounding cloud. In this central volume the temperature varies linearly from edge to centre with the temperature at the edge being that of space at 2.7 Kelvin. Within the volume the temperature decreases to Absolute Zero within the mean molecular path length of hydrogen molecules at this temperature and the pressure of space (approximately $10^{5}$ $\mathrm{kms}$ ). Into the volume are injected hydrogen atoms and molecules through movement originating by intermittent collision of hydrogen atoms and molecules and intermittent absorption of radiation energy from sources situated in all directions with respect to the gas cloud. On entry into the central volume all motions associated with the atoms and molecules of hydrogen progressively ceases. The energy released during this change leaves the volume involved to be absorbed by hydrogen atoms and molecules in the gas cloud. The distribution of matter within the volume at Absolute Zero is related to the variation of energy associated with hydrogen atoms and molecules on approach and entry into the volume. The final result is an enclosed sea of inactive neutrons at a temperature of Absolute Zero.

The conditions of zero spatial movement of matter and zero atom component motion are identified as the conditions associated with Absolute Rest. Bodies of matter in the condition of Absolute Rest exhibit no force of gravitation which involves motion through acceleration. Within the space occupied by matter at Absolute Rest no change occurs and time has no meaning. The matter present under these conditions are identified as neutrons which do not undergo disintegration into a proton and an electron at Absolute Zero and represent the smallest body of matter possible. A body of matter at Absolute Rest does not move nor can be moved through space since there no particle interaction within such a body to induce movement. In this state emission of electrons has ceased and as a consequence no radiation is generated, no radiation can enter or pass through the body and no radiation is reflected by the body. On approaching such body temperature consistently decreases and emitted, transmitted and reflected radiation gradually ceases as electron motion ceases. The sole form of energy possessed by bodies exhibiting Absolute Rest is potential energy since they possess no thermal, gravitational or kinetic energy. These conditions do not violate the principle that energy cannot be created or destroyed.
It is further postulated that neutrons in the outermost regions of a volume containing matter at Absolute Zero can be activated into motion by interaction with the components of a flux of high energy particulate matter as exemplified by cosmic radiation. Motion of the neutrons at the outer reaches is induced by direct collision producing disintegration into protons and electrons or tangential contact inducing neutron rotation which also leads to electron emission and proton formation. Groups of activated neutrons form atom structures by electron distribution or electron exchange. The former leads to structures involving groups of protons enclosed by a cloud of electrons. This arrangement requires the further postulation of the appearance of a force or forces which counters the Coulomb repulsion of the centralised protons. Electron exchange leads to structures formed by electrons circulating in rings of protons between emission and capture. The rings are arranged as one in each of three dimensions at right angles. The atom structure involves several such constructions of diminishing dimensions placed one within the other [7]. Radiation emission is the result of electron motion in both cases. The onset of the formation of atomic structures leads to the conversion of potential energy into kinetic and radiant energy. These interactions only take place at the surface of the volume of space containing matter at Absolute Rest eventually giving rise to an enclosing flare of reacting neutrons which becomes an atmosphere. Activated matter will, in turn, activate other neutrons by the emission of particles and the process proceeds from the outside towards the interior of the region of Absolute Rest. The structure of an activated body at Absolute Rest is one whose surface is entirely covered with a flare at high temperature resulting from of the release of the potential energy as radiant and kinetic energy. The properties of the region detailed above mean that radiant energy only travels outward into space and neutrons at the interior remain motionless at Absolute Zero.

A group of bodies known as pulsars exist alongside stars and are identified by regular intermittant emissions of pulses of radiation such as gamma-, $\mathrm{X}$ - and radio wavelength radiation. Others display a pulsing magnetic field. These bodies are presently considered to be the last stage of decay of a star and to be composed entirely of neutrons. The concept above identifies all of these radiant emissions as originating from the nuclear fusion, nuclear fission and accelerated charged particles forming at the initiation of the activation of a region at Absolute Rest. The pulsing effect is the result of the flare region moving over the boundary surface of the zone at Absolute Rest as expansion proceeds.

The appearance of motion gives rise to gravitation which will retain any formed elements near to the periphery of the flare and result in the activated region acquiring globular form. The physical depth of the flare atmosphere is initially only a small fraction of the physical dimensions of the body of matter at Absolute Rest and consists of a mass of uncharged and oppositely charged particles and atom structures in constant motion. The difference in masses of protons and electrons mean that the distances travelled by each particle in a given direction after emission and /or collision will be different resulting in directional motion of particles in the flare atmosphere. This produces in a flow of negatively charged particles moving in one direction and positively charged particles moving in the opposite direction. These circulations of charge give rise to magnetic fields. The overall magnetic field of the body will be the resultant of all of these fields with an axis at right angles to the plane in which the resultant motion is occurring. A consequence of these magnetic fields is rotation of the flare. Ejection of sections of the flare into space occurs when the escape velocity of formed atoms or molecules is attained or the centrifugal force of rotation becomes sufficiently high. The sections are composed of formed atoms, active neutrons, protons and electrons and neutrons at and near Absolute Zero temperature. These masses become the planets of a star system.

The progress of activation into the the volume of matter at Absolute Rest is indicated by the colour of the flare. This changes from red to orange and through yellow white to white and finally to blue. At each stage the temperature increases from about 6000 Kelvin to 40,000 Kelvin consistent with the progressive activation 
of increasing numbers of neutrons from Absolute Rest. As the depth of the flare increases the volume of the contained mass decreases in proportion to the increasing force of gravity. From this red stars are stars undergoing initial neutron activation. The effects and products of an activated region of Absolute Rest continue until either the neutrons in the volume of the region are dispersed throughout the immediate and remote space as elements and /or bodies of matter or the condensation of elements and stable solids enclose the original flare. In the latter case the reactions continue until complete and no further energy is emitted. Under these conditions and in the absence of a source of radiant energy the body cools and returns to the state of Absolute Rest.

The activation of a region of Absolute Rest produces other observed effects. Microwave radiation with a spectrum peak in the microwave range frequency at $160.2 \mathrm{GHz}$, corresponding to a $1.9 \mathrm{~mm}$ wavelength and with a thermal black body spectrum at a temperature of $2.725 \mathrm{~K}$, is present in space [8]. The origin of this radiation is the electron motions in the flare regions of bodies changing from the state of Absolute Rest to observable motion. The components any groups of repulsive particulate matter exhibit mutual rejection and tend to move apart. This motion will be disturbed by the appearance of a similarly charged particle between any two of the particles as will the disappearance of one of the particles from between any two particles. The activation of the zone of matter at Absolute Rest introduces emission of radiant energy, emission of particulate matter and a progressive increase in gravitation related to forming mass of elements. These changes disturb any surrounding bodies of matter existing above Absolute Rest. The combined effect of activation of several zones of Absolute Rest is the movement apart of the bodies. This resultant of effect is observed in relation to the motion of nebulae.

\section{Formation of the sun and planets of the so- lar system}

The Sun represents the activation of a region of Absolute Rest whose centre is composed of neutrons at Absolute Zero enclosed at the outermost surface by a flare of activated neutrons. The planets and the asteroid belt of the Solar system originated as ejected volumes of the activated body at Absolute Rest. The atom matter which formed in the flare became closed around neutrons at Absolute Zero by the gravity associated with the section of the flare and solidified. The gaseous outer planets of the Solar System are representative of the ejection of a masses of matter originating at the early stages of flare activation formed from a limited amount of low atomic mass elements and related compounds producing a central mass enclosing neutrons at Absolute Rest. These bodies moved to the outer reaches of the initial hydrogen gas cloud under the low value gravitation associated with the early age of flare activation and collected hydrogen from the intergalactic cloud during the movement. These are the gas planets at the outer regions of the Solar System planets and are the oldest planets in the system. The solid planets are composed an outer shell of solid elements and an inner shell of liquid elements and compounds surrounding the section of flare involved plus a core of neutrons at Absolute Zero. The solid planets are younger than the gas planets. The presence of substantial solid matter gave increased gravity of these planets which resulted in the observed present day orbits. The planet Mercury is therefore the youngest plant in the Solar System.

In the Solar system the flux of particles with an energy value of $10^{18} \mathrm{ev}$ or greater is of the order of 1.0 particle $\mathrm{km}^{-2} \mathrm{yr}^{-1}$. The flux of particles with an energy value of $10^{11}$ ev equals $10^{9}$ particles $\mathrm{km}^{-2} \mathrm{sec}^{-1}$ [9], [10]. These values allow an estimate of the age of the Sun. The latter has a surface area of the $5.98 \times 10^{12}$ $\mathrm{km}^{2}$. The first centimetre cubed of this surface has a volume of $8.3 \times 10^{8} \mathrm{~cm}^{3}$. The number neutrons in $1 \mathrm{~cm}^{3}$ of hydrogen is 5.4 $\mathrm{x} 10^{19}$. The total number of neutrons in first $\mathrm{cm}^{3}$ of this volume $=$ $8.3 \times 10^{8} \times 5.4 \times 10^{19}=4.48 \times 10^{28}$. From this the activation of 1 $\mathrm{cm}$ depth of the entire surface of the region of Absolute Rest giving rise to the Sun would take place in approximately $7.6 \times 10^{15}$ years.

Several observations support the above proposals. The above Solar structure accounts for the low value of the solar angular momentum due to the lack of motion of the majority of mass particles within the volume of the Sun. Only the onset of atom formation in the flare gives rise to moving mass. This represents only a fraction of the volume of the Sun. The momentum of the planets is the result of higher mass. It is presently considered that the observed deflection of light by the Sun is an effect of solar gravity. Using Newtonian concepts the deflection of light has a calculated value of 0.87 arc secs and the Einstein concept of distortion of space by gravity produces a value of 1.75 arc secs. A range of measured values is obtained in practice from 0.87 arc seconds to 1.98 arc seconds [11], [12]. The observed deflection of light by the Sun is a therefore a refractive index effect of the flare atmosphere of the Sun. The range of values arises due to non-uniformity of this atmosphere.

\section{The structure of the earth}

The Earth was formed as described above and has an inner core consisting of a sphere of neutrons at Absolute Zero and a flare region in which elements are formed and a solid surface.

The reactions in the flare are the source of the heat generated and the production of elements. Surrounding the flare is the mantle which is a region where elements react to give compounds. The mantle is enclosed by a solidified crust of elements and compounds. The presence of particular elements in any given region of the crust is entirely random as observed by geological studies. Radiation thermal or otherwise travels only outwards from the flare region. In the Earth and other similarly constructed cosmic bodies gravity will decrease to near zero at the edge of the flare region.

Various observations support the above Earth structure. It is presently concluded from observations of seismic wave transmissions that the Earth has an inner core which is solid and an outer core which is liquid on the basis that shear waves are not transmitted by either liquids or gases [13]. The structure above would give the same results. The condition of Absolute Rest produces behaviour similar to the limited atom movement within a solid. The nontransmission of shear waves by the outer core would be expected as the outer core is a nuclear gas which not transmit shear waves. The first isotope formed from hydrogen is the helium-3 isotope. This isotope is rare at the surface of the Earth but occurs in much greater abundance in basalt rock originating from deep within the mantle and a plume of this isotope has been discovered in the Pacific Ocean [14]. This supports the proposal that the outer core of the Earth is the flare region in which atoms are still forming. A discrepancy exists with respect to the value of the density of the material forming the Earth. The mean density has a value of 5.53 grams $\mathrm{ml}^{-3}$. The measured value of the density near the surface is 2.65 grams $\mathrm{ml}^{-3}$. These observations indicate that the density is not uniform and that regions of high density exist beneath the solid crust. Elements formed in the flare region condense at the flare-mantle boundary. The temperature in this region is of the order of $3000^{\circ} \mathrm{K}$. At such temperatures all known chemical compounds would be thermally dissociated into elements, with the exception of carbon dioxide, carbon monoxide and water. Most elements, particularly metals, have a greater density than any of their compounds. For example the element iron has a density of $7.86 \mathrm{gm} \mathrm{ml}^{-3}$ whereas few of the compounds of iron attain this density. Thus the molten mixture of elements in the boundary region of the flare and mantle have a mean density higher than the near surface density. These elements are transported to cooler 
regions of the mantle by thermal convection where they react chemically forming the less dense compounds in the mantle and the crust leading to the density variations observed. Continued rotation of the Earth would eventually cease through collisions with material in space in which the angular momentum of the Earth is transferred to the material involved. The planet will also possess a magnetic field for reasons discussed previously. The mechanism of continuing rotation is the same as that described above which induced rotation into the Solar flare initially. At the flare-mantle boundary the moving flare will encounter friction with the formed atoms. This produces and continues the rotation of the planet.

\section{Discussion}

The Newtonian definition of a body of matter at Absolute Rest assumes that the existence of such a body can be detected by "particular properties, causes and effects" [15]. The first indication of the presence of such a body will be a fall in temperature accompanied by a decrease in the force of gravity of the surrounding space. As a body exhibiting Absolute Rest is approached the motion of electrons giving rise to and the propagation of radiation progressively diminishes. The existence of matter at Absolute Rest and the properties assigned to matter in this state makes the proposal known as Olber's Paradox no longer relevant. This arises as the result of the intervention of such matter in any sight line from Earth to any star will make detection of light from the star impossible.

\section{References}

[1] F. Hoyle. (1948). A New Model for the Expanding Universe MNRAS 108 372-381 http://dx.doi.org/10.1093/mnras/108.5.372

[2] S.S. Holt, C.L. Bennett, V. Trimble, (Editors), the First Three Minutes, American. Institute of. Physics, New York, 1991.

[3] M.Woolfson M. (2000). The origin and Evolution of the Solar System. Astro. \& Geophys. 41 (1) 1.12- 1.19

[4] N. Bohr (1913). Philosophical Magazine.261-25 http://dx.doi.org/10.1080/14786441308634955.

[5] A. Einstein. (1907) on the relativity priciple and the conslusions drawn from it. Jahrbuch der Radioaktivitat. 4 411-462

[6] K. Schwarzschild (1916) "Über das Gravitationsfeld eines Massenpunktes nach der Einsteinschen Theorie", Sitzungsberichte der Deutschen Akademie der Wissenschaften zu Berlin, Klasse fur Mathematik, Physik, und Technik 189-198.

[7] D.S. Robertson, D.S. (1994). Speculation on the nature of the atom. Spec. Sci. Tech., 17, No. 2, 141-158.

[8] Penzias, A.A. Wilson, R.W. (1965). "A Measurement of Excess Antenna Temperature at $4080 \mathrm{Mc} / \mathrm{s}$ ". Astrophys J. 142: 419-421. http://dx.doi.org/10.1086/148307.

[9] S. Swordy. (2001)The energy spectra and anisotropies of cosmic rays. Space Science Reviews 99, 85-94. http://dx.doi.org/10.1023/A:1013828611730.

[10] Sokolsky, P. (1989). Introduction to Ultrahigh Energy Cosmic Ray Physics. Figure 2.2 Addision-Wesley Publishing Company. Redwood City:

[11] A. Einstein. (1911). Onthe influence of gravitation on the propagation of light Annalen der Physik 35 898-908 http://dx.doi.org/10.1002/andp.19113401005.

[12] Kennefick D. (2009) Testing relativity from the 1919 eclipse - a question of bias. Physics Today March 2009 37-42 http://dx.doi.org/10.1063/1.3099578.

[13] B.A. Bolt, Inside the Earth. Freeman and Co., New York, 1982.

[14] WOCE Pacific Program, WHIO Research 1998.

[15] Newton, I. Principia. Unversity of California Press. 1934. 